\title{
A family with hypothyroidism caused by fatty acid synthase and apolipoprotein $B$ receptor mutations
}

\author{
JIANHUA SUN ${ }^{1}$, LIZHI SUN ${ }^{2}$, WEIJIE CHEN ${ }^{3}$, XIAO YIN ${ }^{3}$, YONG LU $^{3}$ and QIANG JIANG ${ }^{3}$ \\ ${ }^{1}$ Department of Oncology, The Second Hospital of Shandong University, Jinan, Shandong 250033; \\ ${ }^{2}$ Central Laboratory, Jinan Central Hospital Affiliated to Shandong University; ${ }^{3}$ Department of Endocrinology, \\ Jinan Central Hospital Affiliated to Shandong University, Jinan, Shandong 250013, P.R. China
}

Received February 2, 2018; Accepted August 15, 2018

DOI: $10.3892 / \mathrm{mmr} .2018 .9499$

\begin{abstract}
Hypothyroidism is a disease with a genetic component. The present study aimed to identify the potential causative gene mutation in a family with hypothyroidism and to investigate its potential pathology. DNA was extracted from the affected individual and his parents, maternal aunt and maternal grandmother. Whole exome sequencing was used to examine their exomes. The potential causative genes that may have an autosomal dominant mode of inheritance were selected after variant calling and filtering. Bioinformatics analysis was utilized to predict the deleteriousness of the identified variants, and multiple sequence alignment and conserved protein domain analyses were performed using online software. Finally, Sanger sequencing was used to validate the identified variants. In the present study, a total of 50 variants were screened based on the autosomal dominant mode of inheritance. Two variants, the fatty acid synthase (FASN) and apolipoprotein B receptor (APOBR) genes, were further analyzed, as they were highly associated with hypothyroidism. Genotyping results revealed that two mutations, c.G7192T (p.A2398S) in the FASN gene and c.C1883G (p.T628R) in the APOBR gene, were fully co-segregated with established hypothyroidism phenotypes in the family. These mutations were located in the conserved $\alpha / \beta$-hydrolase fold and $\mathrm{Na}^{+} / \mathrm{Ca}^{2+}$ exchanger superfamily domain of FASN and APOBR, respectively. In conclusion, the present study demonstrated that the FASN c.G7192T and APOBR c.C1883G mutations may be the potential causative variants in this Chinese hypothyroidism pedigree.
\end{abstract}

Correspondence to: Dr Qiang Jiang, Department of Endocrinology, Jinan Central Hospital Affiliated to Shandong University, 105 Jiefang Road, Jinan, Shandong 250013, P.R. China

E-mail: jiangqiangjinan@sina.com

Abbreviations: APOBR, apolipoprotein B receptor; FASN, fatty acid synthase; SNVs, single nucleotide variants; WES, whole exome sequencing

Key words: WES, hypothyroidism, FASN, APOBR

\section{Introduction}

Thyroid hormones (triiodothyronine and thyroxine) are major regulators of diverse metabolic pathways via interactions with thyroid hormone nuclear receptors in various tissues (1-3). Maintaining thyroid hormone levels is essential for development and metabolism. Hypothyroidism, one of several thyroid diseases, is a pathological state where thyroid hormone levels are decreased systemically or locally in one or more tissues. The prevalence of hypothyroidism is sizeable and ranges between $3-18 \%$ in the adult population, with women, elderly persons and populations with iodine deficiency or excess being more often affected $(4,5)$. Generally, hypothyroidism is diagnosed either in the subclinical or clinical form. Congenital hypothyroidism, if not treated, may lead to severe and irreversible mental retardation (6).

Hypothyroidism is associated with various symptoms, including cold sensitivity, fatigue and lethargy, cognitive dysfunction and delayed growth; these symptoms can be accompanied by distinct signs of tachycardia, weight loss and attention deficit-hyperactivity disorder $(7,8)$. Two of the main characteristics of hypothyroidism are the marked impairment of lipid metabolism and dyslipidemia $(9,10)$. Furthermore, hypercholesterolemia induces increased concentrations of total and low-density lipoprotein cholesterol (11), which will affect normal metabolism. At present, the genetic mechanism implicated in the pathogenesis of hypothyroidism remains poorly understood. It has been reported that mutations in the NK2 homeobox 1 and forkhead box E1 (FOXE1) genes cause hypothyroidism $(12,13)$. In addition, a mutation in immunoglobulin superfamily member 1 has been revealed to be associated with central hypothyroidism (X-linked syndrome) (14). Loss-of-function mutations in thyroglobulin, paired box 8 , thyroid-stimulating hormone receptor, FOXE1, NK2 homeobox (NKX2)-1 and NKX2-5 genes are also associated with inherited congenital hypothyroidism $(15,16)$. Therefore, identifying candidate gene mutations may be helpful in understanding the pathology of hypothyroidism.

Whole exome sequencing (WES) is a useful tool for exploring the genetic mechanism of different diseases (17-19). The present study investigated a Chinese hypothyroidism pedigree, which included an affected 
proband, mother and maternal grandmother; other relatives were unaffected. The results indicated that the c.G7192T (p.A2398S) mutation in fatty acid synthase (FASN) and the c.C1883G (p.T628R) mutation in apolipoprotein B receptor (APOBR) may be the most likely causes of the disease. These results on the inheritance of mutant genes may provide novel information regarding the pathological mechanism underlying hypothyroidism.

\section{Materials and methods}

Subjects and family members. The present study examined a three-generation hypothyroidism pedigree residing in China. The proband (IV:1) was a 3.9-year-old boy who was diagnosed with hypothyroidism. Furthermore, his mother (III:2) and maternal grandmother (II:3) also had hypothyroidism, and all of them shared common characteristics (Fig. 1). In addition, family members I:1 and II:1 were diagnosed with subclinical hypothyroidism, and II:5 was a probable case. The father, maternal aunt and paternal grandparents of the proband were unaffected. Therefore, it was hypothesized that the etiology of hypothyroidism in this family had an autosomal dominant mode of inheritance. Therefore, the proband, along with his parents, maternal aunt and maternal grandmother were enrolled for whole exome sequencing (Fig. 1). Written informed consent was obtained from all individuals enrolled in this study. In addition, the present study was approved by the ethical approval committee of Jinan Central Hospital Affiliated to Shandong University (2016-053-01; Jinan, China).

Exome sequencing and variant calling. Venous blood was obtained from the affected individuals (IV:1, III:2 and II:3) and two unaffected individuals (III:1 and III3) in the hypothyroidism pedigree. The collected blood was stored in EDTA, followed by DNA extraction using a kit (Tiangen Biotech Co., Ltd., Beijing, China). The Agilent SureSelect Human All Exon 50Mb Exon kit (Agilent Technologies, Inc., Santa Clara, CA, USA) was used to perform exome target enrichment of quantified genomic DNA. The amplification process was performed under the following conditions: $15 \mathrm{~min}$ at $95^{\circ} \mathrm{C}$ followed by 40 cycles of $10 \mathrm{sec}$ at $95^{\circ} \mathrm{C}, 30 \mathrm{sec}$ at $55^{\circ} \mathrm{C}, 32 \mathrm{sec}$ at $72^{\circ} \mathrm{C}$, and $15 \mathrm{sec}$ at $95^{\circ} \mathrm{C}, 60 \mathrm{sec}$ at $60^{\circ} \mathrm{C}, 15 \mathrm{sec}$ extension at $95^{\circ} \mathrm{C}$. The Illumina HiSeq 4000 Sequencer (Illumina, San Diego, CA, USA) was utilized for WES. Sequencing reads that had paired-ends, were 200-bp long and had a mean coverage of $100 \mathrm{x}$ were generated for each sample. After filtering out adapter sequences, and contaminated and low quality reads, the clean paired reads were then mapped to the reference human genome sequence, hg19 (20), using the Burrows-Wheeler alignment tool, generating the sequence alignment/map file. Picard software program (version 1.07) was used to mark and remove polymerase chain reaction (PCR) duplicate reads. MuTect (version 1.1.4) and Genome Analysis Toolkit software (version 3.1) (21,22) were used to identify single nucleotide variants ( $\mathrm{SNVs}$ ), deletions and insertions throughout the genome.

Variant filtering. To identify potential candidate genes, the variants were annotated in a systematic manner. Variant information was annotated using various genetic variation databases by the program ANNOVAR (23). From the reported variant frequencies, variants with a minor allele frequency $>0.01$ in the 1000 Genomes Project (https://www. nature.com/articles/nature15393) were excluded. Based on the variant location within genes, higher priority was given to the variants in the coding region and variants that altered the coding sequence (nonsynonymous variants) were identified. The deleteriousness of identified variants was then predicted by bioinformatics analysis [e.g. Oncotator v1.5.3.0, sift (http://sift.jcvi.org/) and polyphen (http://genetics.bwh.harvard.edu/pph2/)], and the harmful nonsynonymous variants were obtained. The eligible nonsynonymous variants were identified using the following filtering parameter criteria in the Genome Analysis Toolkit software (v3.1) (https://software.broadinstitute.org/gatk/): QualByDepth $>2.0$, FisherStrand $<60.0$, StrandOddsRatio $<4.0$, RMSMappingQuality (MQ)>40.0, MQRankSumTest>-12.5, ReadPosRankSumTest $>-8.0$. In addition, minor allele frequency $<0.05$ was also included.

In silico analysis. The multiple sequence alignment of FASN and APOBR in different species was performed using an online tool (https://www.ncbi.nlm.nih.gov/tools/cobalt/cobalt. cgi?LINK_LOC=BlastHomeLink). In addition, the Conserved Domain Search Service (http://www.ncbi.nlm.nih. gov/Structure/cdd/wrpsb.cgi) was used to identify the conserved protein domains.

Sanger sequencing. After the systematic filtering, two candidate variants (FASN c.G7192T, p.A2398S and APOBR c.C1883G, p.T628R) were confirmed. Genomic DNA was prepared from the proband, ten family members and twenty additional, unrelated, affected individuals. Primer-Premier 5.0 (Premier Biosoft International, Palo Alto, CA, USA) was used to design oligonucleotide primer sets for the variants. PCR analyses and Sanger sequencing were then performed. The amplification process was: $15 \mathrm{~min}$ at $95^{\circ} \mathrm{C}$ followed by 40 cycles of $10 \mathrm{sec}$ at $95^{\circ} \mathrm{C}, 30 \mathrm{sec}$ at $55^{\circ} \mathrm{C}, 32 \mathrm{sec}$ at $72^{\circ} \mathrm{C}$, and $15 \mathrm{sec}$ at $95^{\circ} \mathrm{C}, 60 \mathrm{sec}$ at $60^{\circ} \mathrm{C}, 15 \mathrm{sec}$ extension at $95^{\circ} \mathrm{C}$. The PCR products were used for Sanger sequencing.

\section{Results}

Genetic and in silico analysis. Genomic DNA samples from IV:1, III:1, III:2, III:3 and II:3 were analyzed. A total of 50 nonsynonymous variants were identified (Table I). Based on the hypothyroidism in the pedigree and subsequent Sanger sequencing results (Table II), two SNVs, the c.G7192T mutation (a substitution from C to A) in FASN (Chr17: 80037439) and the c.C1883G mutation (a substitution from $\mathrm{C}$ to $\mathrm{G}$ ) in APOBR (Chr16: 28508245), were likely to be associated with the disease. In pedigree individuals, the results of II:3, II:5, III: 2 and IV: 1 showed the c.G7192T mutation in FASN (Chr17: 80037439) and the c.C1883G mutation in APOBR.

In silico analysis. The c.G7192T mutation in FASN resulted in a protein alteration of p.A2398S. In addition, the c.C1883G mutation in APOBR resulted in a protein alteration of p.T628R. Furthermore, multiple sequence alignment and conserved 


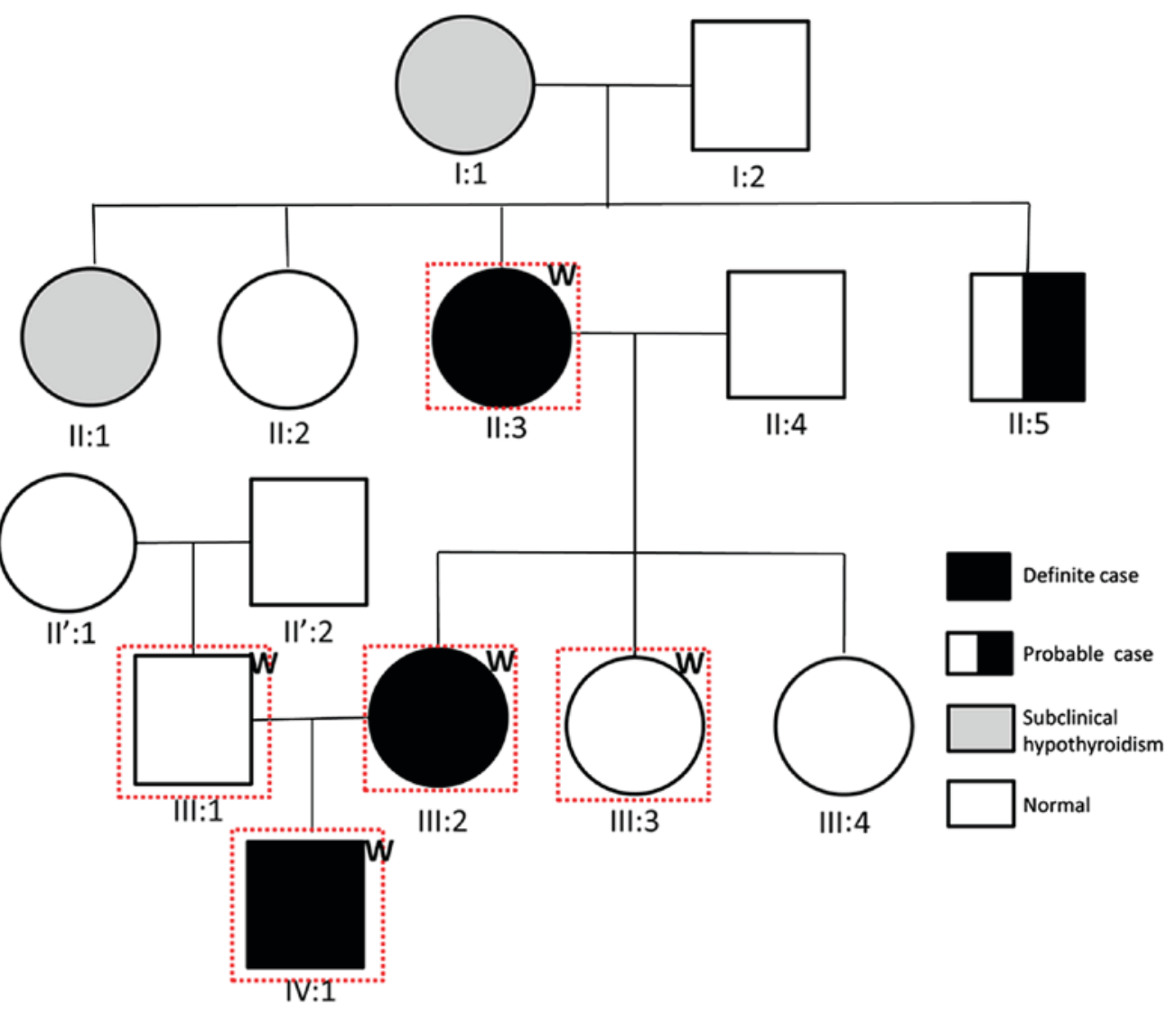

Figure 1. Hypothyroidism pedigree. Exome sequencing was performed on individuals IV:1, III:1, III:2, III:3 and II:3. IV:1 was identified as the proband; I:1 and II:1 were individuals with subclinical hypothyroidism; II:5 was a probable case of hypothyroidism. Circles represent female individuals; squares represent male individuals. W, whole exome sequencing.

A

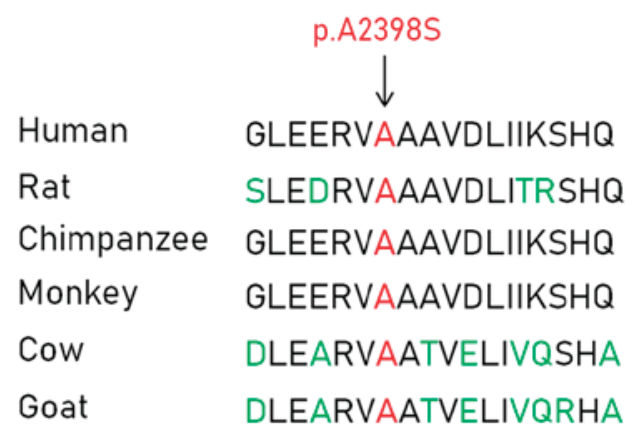

B

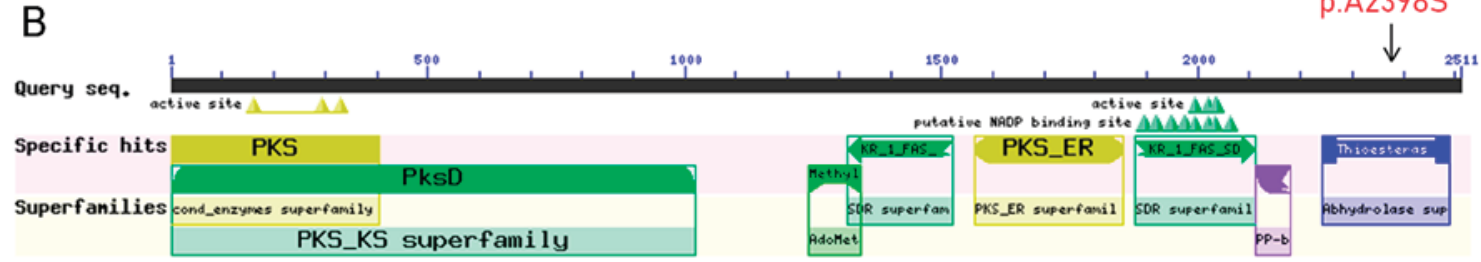

Figure 2. (A) Affected amino acid residue was highly conserved between different species. (B) Conserved domains in fatty acid synthase.

protein domain analyses indicated that the amino acid at position 2,398 in the FASN protein sequence was located in the $\alpha / \beta$-hydrolase fold, and it was highly conserved across several species, including humans, rats, chimpanzees, monkeys, cows and goats (Fig. 2). Furthermore, the amino acid at position 628 in the APOBR protein sequence was located in the $\mathrm{Na}^{+} / \mathrm{Ca}^{2+}$ exchanger superfamily, and it was highly conserved across several species, including humans, monkeys, camels, chimpanzees, pigs and rats (Fig. 3).

Sanger sequencing of the candidate causative variants. In order to further confirm the c.G7192T and c.C1883G mutations in hypothyroidism, Sanger sequencing was performed on the 11 family members and on 20 additional, unrelated individuals 
Table I. A total of 50 nonsynonymous variants in the pedigree.

\begin{tabular}{|c|c|c|c|c|c|c|}
\hline Chr & Start & End & Ref & Alt & Gene.refGene & DbSNP138 \\
\hline 1 & 16057042 & 16057042 & G & A & PLEKHM2 & . \\
\hline 1 & 23238971 & 23238971 & G & A & ЕРНB2 & . \\
\hline 2 & 167760021 & 167760021 & A & $\mathrm{G}$ & XIRP2 & . \\
\hline 2 & 169801131 & 169801131 & $\mathrm{G}$ & $\mathrm{A}$ & ABCB 11 & rs118109635 \\
\hline 2 & 172409920 & 172409920 & $\mathrm{~T}$ & $\mathrm{C}$ & CYBRD1 & rs16859487 \\
\hline 2 & 175618404 & 175618404 & $\mathrm{C}$ & $\mathrm{T}$ & CHRNA1 & . \\
\hline 2 & 179300979 & 179300979 & A & $\mathrm{T}$ & PRKRA & rs77419724 \\
\hline 2 & 179597657 & 179597657 & $\mathrm{~T}$ & $\mathrm{G}$ & TTN & . \\
\hline 2 & 179632619 & 179632619 & $\mathrm{C}$ & $\mathrm{T}$ & TTN & rs141258018 \\
\hline 2 & 204304489 & 204304489 & G & $\mathrm{T}$ & RAPH1 & rs191393494 \\
\hline 2 & 234869499 & 234869499 & G & $\mathrm{C}$ & TRPM8 & . \\
\hline 2 & 238732983 & 238732983 & G & $\mathrm{T}$ & RBM44 & . \\
\hline 3 & 13670739 & 13670739 & G & A & FBLN2 & rs201340643 \\
\hline 3 & 38087142 & 38087142 & G & A & DLEC1 & rs 117463277 \\
\hline 3 & 49701035 & 49701035 & G & A & BSN & rs141950704 \\
\hline 3 & 56597830 & 56597830 & G & A & CCDC66 & rs146224729 \\
\hline 4 & 74363387 & 74363387 & A & $\mathrm{T}$ & AFM & rs2276444 \\
\hline 6 & 90578678 & 90578678 & A & $\mathrm{C}$ & CASP8AP2 & . \\
\hline 6 & 168294583 & 168294583 & G & A & MLLT4 & rs150936076 \\
\hline 6 & 170886768 & 170886768 & $\mathrm{C}$ & $\mathrm{G}$ & PDCD2 & rs140493653 \\
\hline 9 & 140323749 & 140323749 & $\mathrm{C}$ & $\mathrm{T}$ & NOXA1 & rs201388549 \\
\hline 10 & 86132217 & 86132217 & G & $\mathrm{T}$ & CCSER2 & . \\
\hline 11 & 3249828 & 3249828 & $\mathrm{C}$ & $\mathrm{T}$ & MRGPRE & rs200334859 \\
\hline 11 & 3681054 & 3681054 & G & A & ART1 & rs2280133 \\
\hline 11 & 3744621 & 3744621 & $\mathrm{C}$ & $\mathrm{T}$ & NUP98 & rs148384795 \\
\hline 11 & 4411560 & 4411560 & A & $\mathrm{C}$ & TRIM21 & . \\
\hline 11 & 44069816 & 44069816 & G & A & ACCSL & rs182257970 \\
\hline 11 & 58605757 & 58605757 & $\mathrm{C}$ & $\mathrm{T}$ & GLYATL2 & . \\
\hline 11 & 62381857 & 62381857 & G & A & ROM1 & . \\
\hline 11 & 68748268 & 68748268 & A & $\mathrm{G}$ & MRGPRD & rs74390416 \\
\hline 11 & 73067275 & 73067275 & $\mathrm{C}$ & $\mathrm{T}$ & ARHGEF17 & . \\
\hline 11 & 77838424 & 77838424 & $\mathrm{~T}$ & $\mathrm{C}$ & ALG8 & rs138293432 \\
\hline 11 & 93754643 & 93754643 & $\mathrm{~T}$ & A & HEPHL1 & rs192979315 \\
\hline 13 & 96242562 & 96242562 & $\mathrm{~T}$ & $\mathrm{G}$ & DZIP1 & . \\
\hline 15 & 65490592 & 65490592 & $\mathrm{C}$ & $\mathrm{T}$ & CILP & rs148582730 \\
\hline 16 & 28508245 & 28508245 & $\mathrm{C}$ & $\mathrm{G}$ & APOBR & rs 13306186 \\
\hline 16 & 28948654 & 28948654 & $\mathrm{~T}$ & $\mathrm{C}$ & CD19 & . \\
\hline 17 & 4076694 & 4076694 & A & $\mathrm{G}$ & ANKFY1 & . \\
\hline 17 & 4856390 & 4856390 & G & $\mathrm{C}$ & ENO3 & rs143945974 \\
\hline 17 & 10312678 & 10312678 & A & $\mathrm{T}$ & MYH8 & rs151091483 \\
\hline 17 & 19246867 & 19246867 & $\mathrm{~T}$ & $\mathrm{C}$ & B9D1 & rs7221577 \\
\hline 17 & 79167744 & 79167744 & $\mathrm{C}$ & $\mathrm{T}$ & CEP131 & rs138784674 \\
\hline 17 & 80037439 & 80037439 & $\mathrm{C}$ & A & FASN & rs200842352 \\
\hline 18 & 3094191 & 3094191 & $\mathrm{C}$ & $\mathrm{T}$ & MYOM1 & rs149588924 \\
\hline 20 & 76700 & 76700 & G & A & DEFB125 & rs 116934569 \\
\hline 20 & 60575227 & 60575227 & $\mathrm{C}$ & A & TAF4 & . \\
\hline 20 & 60892518 & 60892518 & $\mathrm{C}$ & $\mathrm{T}$ & LAMA5 & rs200632605 \\
\hline 20 & 61167658 & 61167658 & $\mathrm{C}$ & $\mathrm{G}$ & MIR1-1HG & rs145416632 \\
\hline 21 & 34951831 & 34951831 & $\mathrm{C}$ & A & DONSON & rs190773441 \\
\hline 21 & 40568847 & 40568847 & $\mathrm{~T}$ & $\mathrm{C}$ & BRWD1 & rs73357824 \\
\hline
\end{tabular}

Alt, alteration allele; Chr, chromosome; Ref, reference allele; rs, accession number in single nucleotide polymorphism database Build 138 (https://www.ncbi.nlm.nih.gov/projects/SNP/snp_summary.cgi?view+summary=view+summary\&build_id=138). 
Table II. Sanger sequencing validation results.

\begin{tabular}{|c|c|c|c|c|c|}
\hline Individuals & CHRNA1 & ABCB11 & APOBR & FASN & TRPM8 \\
\hline \multicolumn{6}{|c|}{ Affected sporadic individuals } \\
\hline 1 & GG & $\mathrm{CC}$ & $\mathrm{CC}$ & $\mathrm{CC}$ & GG \\
\hline 2 & GG & $\mathrm{CC}$ & $\mathrm{CC}$ & $\mathrm{CC}$ & GG \\
\hline 3 & GG & $\mathrm{CC}$ & $\mathrm{CC}$ & $\mathrm{CC}$ & GG \\
\hline 4 & GG & $\mathrm{CC}$ & $\mathrm{CC}$ & $\mathrm{CC}$ & GG \\
\hline 5 & GG & $\mathrm{CC}$ & $\mathrm{CC}$ & $\mathrm{CC}$ & GG \\
\hline 6 & GG & $\mathrm{CC}$ & $\mathrm{CC}$ & $\mathrm{CC}$ & GG \\
\hline 7 & GG & $\mathrm{CC}$ & $\mathrm{CC}$ & $\mathrm{CC}$ & GG \\
\hline 8 & GG & $\mathrm{CC}$ & $\mathrm{CC}$ & $\mathrm{CC}$ & GG \\
\hline 9 & GG & $\mathrm{CC}$ & $\mathrm{CC}$ & $\mathrm{CC}$ & GG \\
\hline 10 & GG & $\mathrm{CC}$ & $\mathrm{CC}$ & $\mathrm{CC}$ & GG \\
\hline 11 & GG & $\mathrm{CC}$ & $\mathrm{CC}$ & $\mathrm{CC}$ & GG \\
\hline 12 & GG & $\mathrm{CC}$ & $\mathrm{CC}$ & $\mathrm{CC}$ & GG \\
\hline 13 & GG & $\mathrm{CC}$ & $\mathrm{CC}$ & $\mathrm{CC}$ & GG \\
\hline 14 & GG & $\mathrm{CC}$ & $\mathrm{CC}$ & $\mathrm{CC}$ & GG \\
\hline 15 & GG & $\mathrm{CC}$ & $\mathrm{CC}$ & $\mathrm{CC}$ & GG \\
\hline 16 & GG & $\mathrm{CC}$ & $\mathrm{CC}$ & $\mathrm{CC}$ & GG \\
\hline 17 & GG & $\mathrm{CC}$ & $\mathrm{CC}$ & $\mathrm{CC}$ & GG \\
\hline 18 & GG & $\mathrm{CC}$ & $\mathrm{CC}$ & $\mathrm{CC}$ & GG \\
\hline 19 & GG & $\mathrm{CC}$ & $\mathrm{CC}$ & $\mathrm{CC}$ & GG \\
\hline 20 & GG & $\mathrm{CC}$ & $\mathrm{CC}$ & $\mathrm{CC}$ & GG \\
\hline \multicolumn{6}{|c|}{ Pedigree individuals } \\
\hline III-2 & GG & $\mathrm{CC}$ & $\mathrm{CG}$ & $\mathrm{AC}$ & GG \\
\hline II-1 & GA & $\mathrm{CT}$ & $\mathrm{CC}$ & $\mathrm{CC}$ & $\mathrm{GC}$ \\
\hline II-2 & GG & $\mathrm{CC}$ & $\mathrm{CC}$ & $\mathrm{CC}$ & GG \\
\hline II-1 & GG & $\mathrm{CC}$ & $\mathrm{CC}$ & $\mathrm{CC}$ & GG \\
\hline III-3 & GG & $\mathrm{CC}$ & $\mathrm{CC}$ & $\mathrm{CC}$ & GG \\
\hline III-1 & GA & $\mathrm{CT}$ & $\mathrm{CC}$ & $\mathrm{CC}$ & GC \\
\hline II-5 & GA & $\mathrm{CT}$ & $\mathrm{CG}$ & $\mathrm{AC}$ & $\mathrm{GC}$ \\
\hline II-4 & GG & $\mathrm{CC}$ & $\mathrm{CC}$ & $\mathrm{CC}$ & GG \\
\hline II-3 & GA & $\mathrm{CT}$ & $\mathrm{CG}$ & $\mathrm{AC}$ & $\mathrm{GC}$ \\
\hline I-1 & GG & $\mathrm{CC}$ & $\mathrm{CC}$ & $\mathrm{CC}$ & GG \\
\hline IV-1 & GA & $\mathrm{CT}$ & CG & $\mathrm{AC}$ & GC \\
\hline
\end{tabular}

ABCB11, ATP binding cassette subfamily B member 11; APOBR, apolipoprotein B receptor; CHRNA1, cholinergic receptor nicotinic $\alpha 1$ subunit; FASN, fatty acid synthase; TRPM8, transient receptor potential cation channel subfamily M member 8 .

with primary hypothyroidism. The clinical information of the 20 additional, unrelated individuals with primary hypothyroidism is presented in Table III. The results demonstrated that the c.G7192T mutation in FASN and the c.C1883G mutation in APOBR were fully co-segregated with established hypothyroidism phenotypes in the family recruited for WES (Figs. 4 and 5). Furthermore, the FASN c.G7192T and APOBR c.C1883G mutations were detected in the patient with probable hypothyroidism (II:5). It is worth mentioning that the FASN c.G7192T and APOBR c.C1883G mutations were not observed in individuals with subclinical hypothyroidism (I:1, II:1). In addition, the mutations were not detected in the unrelated, affected individuals with hypothyroidism (date not shown), which supported the hypothesis of genetic heterogeneity of hypothyroidism in this family.

\section{Discussion}

Hypothyroidism is the most common endocrine disorder caused by thyroid hormone deficiency, which can induce metabolic dysfunction (24). At present, the genetic mechanisms underlying hypothyroidism pathogenesis remain poorly understood. It has been reported that the c.1184_1187dup4 mutation in the thyroid peroxidase gene, c.40A $>\mathrm{G}$ and c. $94 \mathrm{G}>\mathrm{A}$ mutations in the thyroid-stimulating hormone $\beta$ gene, p.G488R, p.A649E, p.R885Q, p.I1080T and p.A1206T mutations in the dual oxidase 2 gene, and the p.Y138X mutation in the dual oxidase maturation factor 2 gene are associated with congenital hypothyroidism (25-27). WES technology is an effective method for identifying potential causative genes in disease phenotypes. In the present study, 
A

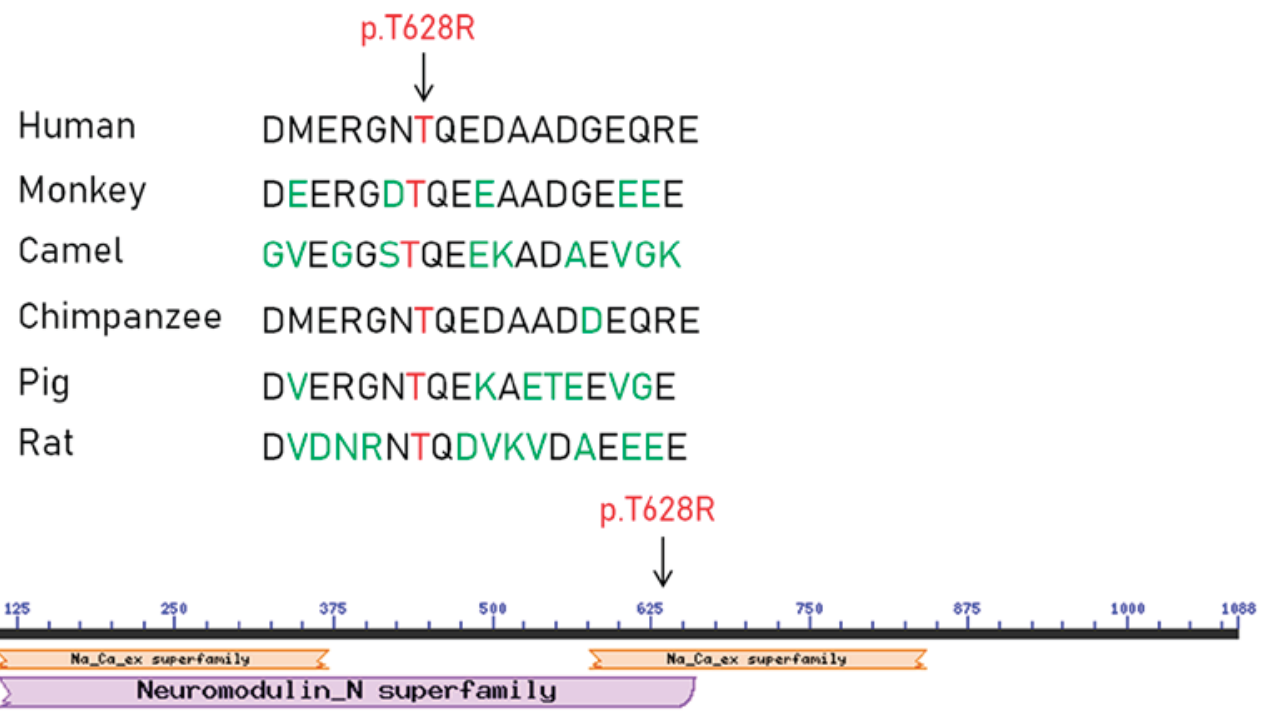

Figure 3. (A) Affected amino acid residue was highly conserved between different species. (B) Conserved domains in apolipoprotein B receptor.

WES was performed to identify potential causative genes in the affected individual, his parents, maternal aunt and maternal grandmother in a hypothyroidism pedigree. Two mutations (c.G7192TT and c.C1883G) in the FASN and APOBR genes, respectively, were revealed to be associated with hypothyroidism. In addition, Sanger sequencing validated these FASN and APOBR mutations in the proband and his mother. Furthermore, the mutations were fully co-segregated with established hypothyroidism phenotypes in the family.

Fatty acid synthesis is a process that entails producing de novo fatty acids from carbohydrate- and amino acid-derived carbon sources. The liver serves a key role in fatty acid modulation. The key enzymes in the lipogenic and lipolytic pathways are regulated by thyroid hormones in the liver and adipose tissues $(28,29)$. Disrupted thyroid hormone levels alter hepatic fatty acid composition (30). In addition, it has been reported that subclinical and clinical hypothyroidism are associated with hepatic steatosis (31). Numerous studies have indicated that hypothyroidism is a risk factor for nonalcoholic fatty liver disease and results in metabolic syndrome (32-34). In addition, the effects of hypothyroidism to enhance lipogenesis is amplified in the presence of physiological concentrations of fatty acids (35). FASN is an enzyme involved in fatty acid biosynthesis (36). In mammals, cells manufacture de novo fatty acids using different pathways, which synthesize fatty acids from acetyl and malonyl esters of CoA that are catalyzed by dimerized FASN (37). Furthermore, fatty acid synthesis is controlled by FASN (38), and the downregulation of FASN protein levels causes markedly decreased regulation of de novo lipogenesis (30). Notably, FASN is downregulated in the livers of patients with hypothyroidism (39). In addition, a microarray analysis revealed that FASN, one of the hepatic target genes, is preferentially activated by triiodothyronine during transition from the hypothyroid state to the euthyroid state (40). The c.G7192T mutation is located in the conserved $\alpha / \beta$-hydrolase fold of FASN and was highly conversed among several diverse species, including humans, rats, chimpanzees,

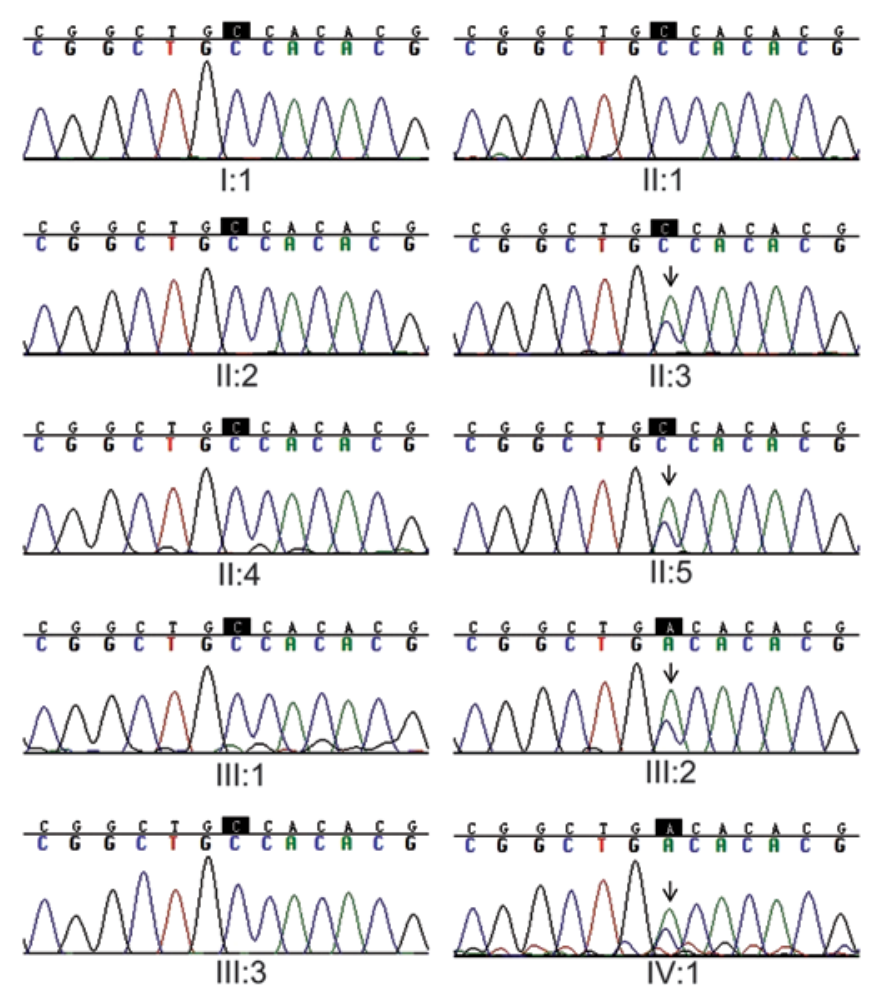

Figure 4. Sanger validation results of fatty acid synthase variants in ten family members of the hypothyroidism pedigree. Arrow represents the mutation site.

monkeys, cows and goats. Therefore, this FASN mutation suggested that FASN may have roles in hypothyroidism.

APOBR is an apolipoprotein E-independent receptor that binds APOB48, allowing cells to uptake postprandial triglyceride-rich lipoproteins, for which APOBR has a high affinity; APOBR functions as a nutritional receptor that provides dietary fatty acids and lipid-soluble vitamins to cells (41). The APOBR protein is distributed in the moieties of plasma triglyceride-rich lipoproteins. It has been reported that APOBR is an important molecule in lipid metabolism, 
Table III. Clinical information of 20 additional, unrelated individuals with primary hypothyroidism.

\begin{tabular}{clccc}
\hline Case & Sex & Age (years) & TSH (mU/l) & FT4 (pmol/l) \\
\hline 1 & Male & 75 & 27.7 & 7.31 \\
2 & Male & 49 & 4.85 & 16.2 \\
3 & Male & 47 & 72 & 0.88 \\
4 & Male & 67 & 9.15 & 10.7 \\
5 & Male & 47 & 1.71 & 15.3 \\
6 & Female & 64 & 3.07 & 15.7 \\
7 & Female & 31 & 6.21 & 12.3 \\
8 & Female & 21 & 5.56 & 11.5 \\
9 & Female & 29 & 1.59 & 10.3 \\
10 & Female & 25 & 5.52 & 13.2 \\
11 & Female & 27 & 4.56 & 12.7 \\
12 & Female & 31 & 3.97 & 16 \\
13 & Female & 32 & 2.57 & 10 \\
14 & Female & 68 & 37 & 7.08 \\
15 & Female & 54 & 5.08 & 11.5 \\
16 & Female & 30 & 57.5 & 7.16 \\
17 & Female & 36 & 4.3 & 12.2 \\
18 & Female & 29 & 0.969 & 11.4 \\
19 & Female & 35 & 9.74 & 12.4 \\
20 & Female & 27 & 4.26 & 11.5 \\
\hline
\end{tabular}

FT4, free thyroxine; TSH, thyroid-stimulating hormone.

and that it primarily serves roles in the sterol transport and metabolism pathways (42). Mutations in the APOBR gene (c.934-960/del and A419P) have been identified as novel hyperlipidemia-associated variants, which may be involved in regulating plasma total cholesterol levels in patients with hypercholesterolemia (43). APOBR is also involved in atherogenesis, which is a lipid metabolism disorder (44). Therefore, it may be hypothesized that the APOBR pathway is a novel therapeutic target, particularly in hypertriglyceridemic patients, such as those with hypothyroidism. In the present study, a mutation (c.C1883G, p.T628R) in the APOBR gene was detected in patients with hypothyroidism. The mutation is located in the conserved $\mathrm{Na}^{+} / \mathrm{Ca}^{2+}$ exchanger domain of APOBR and was highly conversed among different species, including humans, monkeys, camels, chimpanzees, pigs and rats. Therefore, this APOBR mutation may be considered another candidate molecule in the pathogenesis of hypothyroidism.

In conclusion, the findings reported here demonstrated that patients with mutations in FASN (c.G7192T, p.A2398S) and APOBR (c.C1883G, p.T628R) may be predisposed to hypothyroidism. These mutations may disrupt the regulation of fatty acid biosynthesis and lipid metabolism. These findings may reveal the high degree of genetic heterogeneity in hypothyroidism phenotypes. Future work will be performed to improve understanding of this disorder. In addition, there is a limitation of the present study; the identified SNVs, FASN (c.G7192T, p.A2398S) and APOBR (c.C1883G, p.T628R), require validation in animal models of hypothyroidism.

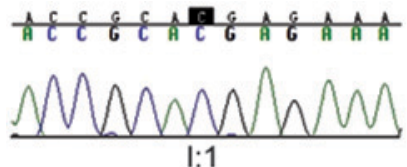

I:1

II:2


III:1

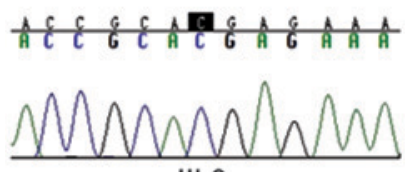

III:3

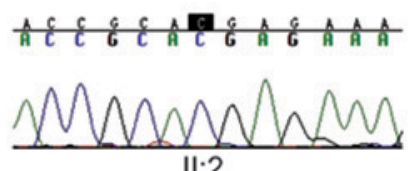

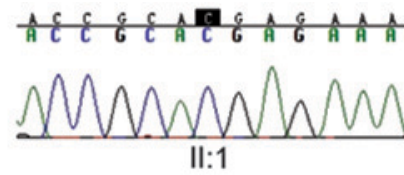
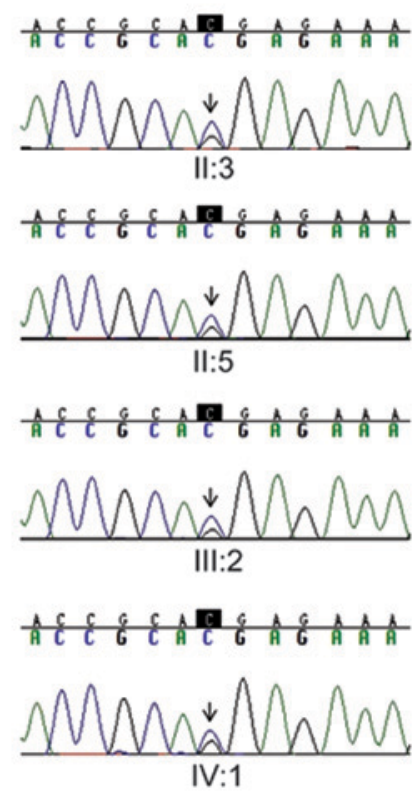

Figure 5. Sanger validation results of apolipoprotein B receptor variants in ten family members of the hypothyroidism pedigree. Arrow represents the mutation site.

\section{Acknowledgements}

Not applicable.

\section{Funding}

Not applicable.

\section{Availability of data and materials}

All data generate or analyzed during the present study are included in this published article.

\section{Authors' contributions}

JS, LS, WC, XY and YL analyzed and interpreted the data. JS was a major contributor in writing the manuscript. QJ designed the project. All authors read and approved the final manuscript.

\section{Ethics approval and consent to participate}

Written informed consent was obtained from all individuals enrolled in this study. In addition, the present study was approved by the ethical approval committee of Jinan Central Hospital Affiliated to Shandong University (2016-053-01).

\section{Patient consent for publication}

Informed written consent was obtained from all subjects. 


\section{Competing interests}

The authors declare that they have no competing interests.

\section{References}

1. Yen PM: Physiological and molecular basis of thyroid hormone action. Physiol Rev 81: 1097-1142, 2001.

2. Zhang $J$ and Lazar MA: The mechanism of action of thyroid hormones. Annu Rev Physiol 62: 439-466, 2000.

3. Song Y, Yao X and Ying H: Thyroid hormone action in metabolic regulation. Protein Cell 2: 358-368, 2011.

4. Virgini V, Baumgartner C, Bischoff T, Haller DM, Frey P, Rosemann T, Collet TH, Sykiotis G, Pitteloud N and Rodondi N: Comment les médecins de famille prennent-ils en charge l'hypthyroïdie infraclinique? Rev Med Suisse 10: 526-529, 2014 (In French).

5. Baumgartner C, Blum MR and Rodondi N: Subclinical hypothyroidism: Summary of evidence in 2014. Swiss Med Wkly 144 w14058, 2014.

6. Fuhrer D, Brix K and Biebermann H: Understanding the healthy thyroid state in 2015. Eur Thyroid J 4 (Suppl 1): S1-S8, 2015.

7. Yu H, Yang Y,Zhang M, Lu H, Zhang J, Wang H and Cianflone K: Thyroid status influence on adiponectin, acylation stimulating protein (ASP) and complement C3 in hyperthyroid and hypothyroid subjects. Nutr Metab(Lond) 3: 13, 2006.

8. Miller LD, McPhie P, Suzuki H, Kato Y, Liu ET and Cheng SY: Multi-tissue gene-expression analysis in a mouse model of thyroid hormone resistance. Genome Biol 5: R31, 2004

9. Yang N, Yao Z, Miao L, Liu J, Gao X, Xu Y and Wang G: Homocysteine diminishes apolipoprotein A-I function and expression in patients with hypothyroidism: A cross-sectional study. Lipids Health Dis 15: 123, 2016.

10. Roberts CG and Ladenson PW: Hypothyroidism. Lancet 363: 793-803, 2004

11. Wang Y, Yu X, Zhao QZ, Zheng S, Qing WJ, Miao CD and Sanjay J: Thyroid dysfunction, either hyper or hypothyroidism, promotes gallstone formation by different mechanisms. J Zhejiang Univ Sci B 17: 515-525, 2016.

12. Veneziano L, Parkinson MH, Mantuano E, Frontali M, Bhatia KP and Giunti P: A novel de novo mutation of the TITF1/NKX2-1 gene causing ataxia, benign hereditary chorea, hypothyroidism and a pituitary mass in a UK family and review of the literature. Cerebellum 13: 588-595, 2014.

13. Mendieta-Zeron H, Jimenez-Rosales A, Perez-Amado CJ and Jimenez-Morales S: FOXE1 mutation screening in a case with cleft lip, hypothyroidism, and thyroid carcinoma: A new syndrome? Case Rep Genet 2017: 6390545, 2017.

14. Sun Y, Bak B, Schoenmakers N, van Trotsenburg AS, Oostdijk W, Voshol P, Cambridge E, White JK, le Tissier P, Gharavy SN, et al: Loss-of-function mutations in IGSF1 cause an X-linked syndrome of central hypothyroidism and testicular enlargement. Nat Genet 44: 1375-1381, 2012.

15. Watanabe Y, Sharwood E, Goodwin B, Creech MK, Hassan HY, Netea MG, Jaeger M, Dumitrescu A, Refetoff S, Huynh T and Weiss RE: A novel mutation in the TG gene (G2322S) causing congenital hypothyroidism in a sudanese family: A case report. BMC Med Genet 19: 69, 2018.

16. Vincenzi M, Camilot M, Ferrarini E, Teofoli F, Venturi G, Gaudino R, Cavarzere P, De Marco G, Agretti P, Dimida A, et al: Identification of a novel pax8 gene sequence variant in four members of the same family: From congenital hypothyroidism with thyroid hypoplasia to mild subclinical hypothyroidism. BMC Endocr Disord 14: 69, 2014.

17. Bamshad MJ, Ng SB, Bigham AW, Tabor HK, Emond MJ, Nickerson DA and Shendure J: Exome sequencing as a tool for mendelian disease gene discovery. Nat Rev Genet 12: 745-755, 2011.

18. Ng SB, Turner EH, Robertson PD, Flygare SD, Bigham AW, Lee C, Shaffer T, Wong M, Bhattacharjee A, Eichler EE, et al: Targeted capture and massively parallel sequencing of 12 human exomes. Nature 461: 272-276, 2009.

19. Wang JL, Yang X, Xia K, Hu ZM, Weng L, Jin X, Jiang H, Zhang P, Shen L, Guo JF, et al: TGM6 identified as a novel causative gene of spinocerebellar ataxias using exome sequencing. Brain 133: 3510-3518, 2010.
20. Li H and Durbin R: Fast and accurate short read alignment with Burrows-Wheeler transform. Bioinformatics 25: 1754-1760, 2009.

21. McKenna A, Hanna M, Banks E, Sivachenko A, Cibulskis K, Kernytsky A, Garimella K, Altshuler D, Gabriel S, Daly M and DePristo MA: The genome analysis toolkit: A mapreduce framework for analyzing next-generation DNA sequencing data. Genome Res 20: 1297-1303, 2010.

22. Cibulskis K, Lawrence MS, Carter SL, Sivachenko A, Jaffe D, Sougnez C, Gabriel S, Meyerson M, Lander ES and Getz G: Sensitive detection of somatic point mutations in impure and heterogeneous cancer samples. Nat Biotechnol 31: 213-219, 2013.

23. Wang K, Li M and Hakonarson H: ANNOVAR: Functional annotation of genetic variants from high-throughput sequencing data. Nucleic Acids Res 38: e164, 2010.

24. Iwen KA, Schroder E and Brabant G: Thyroid hormones and the metabolic syndrome. Eur Thyroid J 2: 83-92, 2013.

25. Cangul H, Aydin BK and Bas F: A homozygous TPO gene duplication (c.1184_1187dup4) causes congenital hypothyroidism in three siblings born to a consanguineous family. J Pediatr Genet 4: 194-198, 2015.

26. Ozhan B, Boz Anlas O, Sarikepe B, Albuz B and Semerci Gunduz N: Congenital central hypothyroidism caused by a novel thyroid-stimulating hormone-beta subunit gene mutation in two siblings. J Clin Res Pediatr Endocrinol 9: 278-282, 2017.

27. Park KJ, Park HK, Kim YJ, Lee KR, Park JH, Park JH, Park HD, Lee SY and Kim JW: DUOX2 mutations are frequently associated with congenital hypothyroidism in the korean population. Ann Lab Med 36: 145-153, 2016.

28. Diamant S, Gorin E and Shafrir E: Enzyme activities related to fatty-acid synthesis in liver and adipose tissue of rats treated with triiodothyronine. Eur J Biochem 26: 553-559, 1972.

29. Oppenheimer JH, Schwartz HL, Lane JT and Thompson MP: Functional relationship of thyroid hormone-induced lipogenesis, lipolysis, and thermogenesis in the rat. J Clin Invest 87: 125-132, 1991.

30. Yao X, Hou S, Zhang D, Xia H, Wang YC, Jiang J, Yin H and Ying $\mathrm{H}$ : Regulation of fatty acid composition and lipid storage by thyroid hormone in mouse liver. Cell Biosci 4: 38, 2014.

31. Ludwig U, Holzner D, Denzer C, Greinert A, Haenle MM, Oeztuerk S, Koenig W, Boehm BO, Mason RA, Kratzer W, et al: Subclinical and clinical hypothyroidism and non-alcoholic fatty liver disease: A cross-sectional study of a random population sample aged 18 to 65 years. BMC Endocr Disord 15: 41, 2015.

32. Amarapurkar D, Kamani P, Patel N, Gupte P, Kumar P, Agal S, Baijal R, Lala S, Chaudhary D and Deshpande A: Prevalence of non-alcoholic fatty liver disease: Population based study. Ann Hepatol 6: 161-163, 2007.

33. Law K and Brunt EM: Nonalcoholic fatty liver disease. Clin Liver Dis 14: 591-604, 2010.

34. Ortiz-Lopez C, Lomonaco R, Orsak B, Finch J, Chang Z, Kochunov VG, Hardies J and Cusi K: Prevalence of prediabetes and diabetes and metabolic profile of patients with nonalcoholic fatty liver disease (NAFLD). Diabetes Care 35: 873-878, 2012

35. Baht HS and Saggerson ED: A tissue-specific increase in lipogenesis in rat brown adipose tissue in hypothyroidism. Biochem $\mathrm{J}$ 251: 553-557, 1988.

36. Rusinek D, Swierniak M, Chmielik E, Kowal M, Kowalska M, Cyplinska R, Czarniecka A, Piglowski W, Korfanty J, Chekan M, et al: BRAFV600E-associated gene expression profile: Early changes in the transcriptome, based on a transgenic mouse model of papillary thyroid carcinoma. PLoS One 10: e0143688, 2015.

37. Smith S: The animal fatty acid synthase: One gene, one polypeptide, seven enzymes. FASEB J 8: 1248-1259, 1994.

38. Suburu J, Shi L, Wu J, Wang S, Samuel M, Thomas MJ, Kock ND, Yang G, Kridel S and Chen YQ: Fatty acid synthase is required for mammary gland development and milk production during lactation. Am J Physiol Endocrinol Metab 306: E1132-E1143, 2014.

39. Blennemann B, Leahy P, Kim TS and Freake HC: Tissue-specific regulation of lipogenic mRNAs by thyroid hormone. Mol Cell Endocrinol 110: 1-8, 1995.

40. Yen PM, Feng X, Flamant F, Chen Y, Walker RL, Weiss RE, Chassande O, Samarut J, Refetoff S and Meltzer PS: Effects of ligand and thyroid hormone receptor isoforms on hepatic gene expression profiles of thyroid hormone receptor knockout mice. EMBO Rep 4: 581-587, 2003. 
41. Brown ML, Yui K, Smith JD, LeBoeuf RC, Weng W, Umeda PK, Li R, Song R, Gianturco SH and Bradley WA: The murine macrophage apoB-48 receptor gene (Apob-48r): Homology to the human receptor. J Lipid Res 43: 1181-1191, 2002.

42. Wang K, Edmondson AC, Li M, Gao F, Qasim AN, Devaney JM, Burnett MS, Waterworth DM, Mooser V, Grant SF, et al: Pathway-wide association study implicates multiple sterol transport and metabolism genes in HDL cholesterol regulation. Front Genet 2: 41, 2011.

43. Fujita Y, Ezura Y, Bujo H, Nakajima T, Takahashi K, Kamimura K, Iino Y, Katayama Y, Saito Y and Emi M: Association of nucleotide variations in the apolipoprotein B48 receptor gene (APOB48R) with hypercholesterolemia. J Hum Genet 50: 203-209, 2005.
44. Brown ML, Ramprasad MP, Umeda PK, Tanaka A, Kobayashi Y, Watanabe T, Shimoyamada H, Kuo WL, Li R, Song R, et al: A macrophage receptor for apolipoprotein B48: Cloning, expression, and atherosclerosis. Proc Natl Acad Sci USA 97: 7488-7493, 2000 .

(i) (9) This work is licensed under a Creative Commons Attribution 4.0 International (CC BY-NC 4.0) License 